CARNETS OE Carnets de géographes

GÉOGRAPHES

5 | 2013

Géographie humanimale

\title{
La corporéité cynégétique
}

Une démarche territoriale entre symbolique locale et spatialité animale

\section{Christophe Baticle}

\section{(2) OpenEdition}

Journals

Édition électronique

URL : http://journals.openedition.org/cdg/1055

DOI : $10.4000 /$ cdg. 1055

ISSN : 2107-7266

Éditeur

UMR 245 - CESSMA

Référence électronique

Christophe Baticle, «La corporéité cynégétique », Carnets de géographes [En ligne], 5 | 2013, mis en ligne le 01 janvier 2013, consulté le 01 mai 2019. URL : http://journals.openedition.org/cdg/1055 ; DOI : $10.4000 /$ cdg. 1055

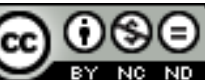

La revue Carnets de géographes est mise à disposition selon les termes de la Licence Creative Commons Attribution - Pas d'Utilisation Commerciale - Pas de Modification 4.0 International. 


\title{
LA CORPOREITE CYNEGETIQUE. Une démarche territoriale entre symbolique locale et spatialité animale
}

\author{
CHRISTOPHE BATICLE \\ Socio-anthropologue \\ Université de Picardie Jules Verne, Amiens. \\ EA Habiter le Monde/UMR-CNRS CURAPP \\ cbaticle@aol.com
}

\begin{abstract}
Résumé
L'exercice de la chasse exige tant une initiation symbolique, relevant du social, qu'un apprentissage par corps qui se trouve médiatisé au travers du territoire. Si ce n'est que la finalité reste ici la ruse à l'égard du gibier, lequel fait aussi montre de sa propre logique comportementale. Ainsi, dans l'observation des stratégies cynégétiques, on perçoit que le jeu avec l'animal ne peut pas être cantonné au statut de reflet des représentations sociales, dont pour autant il fait également l'objet. De surcroit, les attitudes de la faune sauvage jouent comme de puissants vecteurs de l'agir cynégétique. Bonnes à penser, les postures animales provoquent des interprétations humaines qui interrogent le débat sur le déterminisme social lorsque se trouve introduit un tiers-acteur entre l'homme et son espace : un gibier obligeant à une forme de réflexivité.
\end{abstract}

\begin{abstract}
The practise of hunting requires a symbolic initiation linked to the social side of things, as well as an initiation through the body which itself finds its place in the territory. We must keep in mind that the finality here is the crafty tactics you use facing the game, this very game having its own logical behaviour. So while studying the strategies related to hunting, you can easily discover that playing with the animal can't be reduced to the mirror image of social representations. Moreover, the way wild life behaves, acts as powerful vector in the hunting scene. The way animals behave lead to human interpretations that induce questions related to social determinism when a third acting party intervenes between the human being and his territory; a wild animal inducing a reflexivity of some sort.
\end{abstract}

" Les trajets concentriques désignent cet ensemble de déplacements qui marquent un lieu comme central. Les rites d'installation, de reconnaissance, le repérage du voisinage, sont autant de rondes qui constituent le réseau de définition d'un lieu. "

(Sansot et al., $1978: 56)$.

La pratique cynégétique convoque le corps à bien des égards: chasseurs à la billebaude déambulant sur la plaine, nemrods statiques maîtrisant leurs moindres gestes à la corne d'un bois, tireurs dépeçant les pièces de leur tableau après la mise à mort, ou même 
guetteurs perchés à la cime des arbres comme dans le cas des palombières du Sud-ouest hexagonal. L'approche consistant à étudier cette démarche de prédation sous l'angle de ses pratiquants, mais ici entendus en tant que corporéités autant agies qu'agissantes, rencontre plusieurs manières d'appréhender le corps spatialisé, soit en l'occurrence pour nous celui du porteur de fusil, mais encore la spatialité incorporée du territoire de la pratique, à savoir une manière d'habiter la nature sur un mode prédateur spécifique (Baticle, Mazzéi, 2011), le tout orienté par une variable incertaine, la ruse de l'animal chassé.

Il s'agit tout d'abord du corps centre de la perception, et par là moyen de rendre perceptible l'environnement physique. On se situe à ce niveau dans la construction d'un être-là au monde, ici et maintenant (hic et nunc pour reprendre les termes de la phénoménologie), au cours d'un processus ouvert qui implique la présence. Le chasseur de lièvre, par exemple, apprend par corps (et non par cœur, ce qui renverrait à un apprentissage intellectualisé) à se comporter de façon à débusquer le petit mammifère dont il a progressivement intégré certaines manières de faire au sein d'un biotope donné. II est ensuite, ce corps, la matière sensible à une histoire du déchiffrage, aussi bien sociale que culturelle, dont la corporéité est cette fois le réceptacle, un dépositaire grandement involontaire. Le déjà construit domine dans cette seconde manière de concevoir le sens des signes perçus ou au contraire laissés dans le magma de l'imperceptible, du hors-sens. Le même chasseur envisage sa quête du lagomorphe sous le prisme d'une symbolique qui fut prégnante pour les sociétés rurales: le bouquin restant encore aujourd'hui l'animal roi des plaines. Ce faisant, sa prise est élective pour son poursuivant, lui conférant une certaine notoriété parmi ses confrères.

Surtout, l'espace sécant entre cet agir cynégétique (l'extériorisation du corps par l'action) et l'ensemble des dispositions héritées dont le corps devient le siège privilégié (l'intériorisation charnelle d'une socialisation à être chasseur) offre une potentialité heuristique pour proposer une lecture dans un très ancien débat sociologique sur le déterminisme. Afin de résumer succinctement, posons que la dynamique entre individu et société, personnalité et groupe d'appartenance, ne peut se limiter à une détermination univoque de l'un des pôles sur l'autre, ce à quoi la plupart des sociologues adhèrent désormais (Maggi, 2011). Une concession quasi unanime qui se trouve corrélée à la prise en considération d'un tiers acteur dans l'analyse des interactions, soit justement le contexte interactif, la situation, en bref l'environnement dans lequel se déroule la relation entre le Moi et l'Autrui, dont au premier rang ici l'animal. Reste néanmoins que ce milieu, son ambiance, le climat qui y règne, ou l'aire morale (Hall, 1971) dont il relève, nous reporte à l'alternative précédente: soit on estime avoir affaire à un pré-formatage social qui encadre l'interrelation au travers d'une élaboration matérielle emplie de significations socioculturelles, soit on privilégie le possible renouvellement des schèmes perceptifs et interprétatifs à partir d'une configuration physique laissant la place à la réinterprétation. En d'autres termes, les cadres de l'expérience humaine se réalisant dans des espaces significativement socialisés, peut-on attendre du corps placé dans cette forêt de symboles autre chose que des innovations socialement ad hoc. Pour illustrer, la sociologie de l'espace s'est heurtée à ce choix cornélien : tout dispositif spatial reflète bien l'ordre sociétal qui lui a donné naissance et dispose ainsi à un mode de socialisation pré-formaté, alors que réciproquement les usages qu'il est fait de n'importe lequel de ces agencements ne répondent jamais exactement aux attendus de leurs inventeurs, ce qui fait qu'on ne puisse pas incriminer aussi facilement les grands ensembles dans le malaise des dites "banlieues" (Chamboredon, Lemaire, 1970). Espace socialisé et socialisant (Bordreuil, Ostrowetsky, 1984) en quelque-sorte, mais relevant d'une socialisation imprévisible, "l'homme spatial » (Lussault, 2007) s'y meut en 
découvreur du monde et de lui-même, mais équipé d'une boussole dont on ne sait si elle lui donne la direction qui fait sens afin de ne pas "perdre le Nord", ou si elle permet d'imaginer de nouvelles cosmogonies.

Dans ce cadre réflexif, l'apport d'une activité comme la pratique cynégétique tient dans son originalité a priori extra-sociologique. Même s'il est question d'un exercice physique proprement social, certains discours cherchant d'ailleurs à en faire un loisir " comme un autre ", son objet nous éloigne des interactions habituellement étudiées entre acteurs sociaux (ou agents selon le parti-pris théorique adopté). Les adeptes de Diane, sauf à les observer dans leurs seules relations hors action de chasse, sont mus par des logiques comportementales qui constituent des formes d'adaptations culturelles aux attitudes du monde animal, non réductibles donc à l'équation courante des sciences sociales, dans laquelle la symbolisation joue un rôle essentiel. En conséquence, si le nemrod contemporain est loin d'être un sauvage en puissance (Dalla-Bernardina, 2011), il doit fait montre d'une forme d'ensauvagement s'il veut espérer ne pas rentrer systématiquement bredouille. Nous verrons dans un premier temps que cette acquisition incorporée passe par des mécanismes qui empruntent au langage du corps à travers l'espace, mais aussi et dans un second temps qu'une forme langagière directement cynégétique se dégage de cet apprentissage, qu'elle se traduit par des attitudes physiques et par des manières de se mouvoir sur le territoire. En définitive, la médiation de la nature dans ce jeu avec l'animal amène à s'interroger sur une possible renégociation des schèmes perceptifs, et ce via l'introduction d'un " partenaire " (involontaire) qu'il convient d'incorporer dans son schéma de perception, alors que l'on se situe en dehors de toute forme de rationalité humaine et donc a fortiori sociale.

\section{L'apprentissage par corps d'une pratique de nature}

II n'existe pas de chasse au singulier, ce que Marcel Mauss avait déjà suffisamment montré en affirmant que "L'individu ne va pas à la chasse, il va à la chasse au lièvre " (Bromberger, Lenclud, 1982 : 20), et d'ajouter utilement « et non pas à la chasse au lièvre mais à la chasse de tel lièvre, qu'il connaît bien. " Précision essentielle qui nous réinstalle dans la vision d'une faune socialisée par la connaissance quasi chirurgicale que l'on acquière sur ses habitudes de vie. Motif supplémentaire pour poser d'emblée que les pratiques cynégétiques forment un univers où règne la diversité, tant en termes de méthodes, d'instruments de capture, de saisonnalité, qu'en termes d'espèces considérées comme "gibiers ". Vouloir recenser cette variété relève de la gageure, tant les régions apportent pour une seule forme de pratique autant de modalités d'exercice que ne le permettent les spécificités écosystémiques locales, les interdits culturels ou encore la panoplie des situations compétitives dans l'accès aux territoires.

La mise en place d'un "sens pratique " (Bourdieu, 1980) implique pour la chasse que l'on s'attache d'abord à bien relever l'importance de la topographie sociale des lieux, avant de revenir sur les procédures d'apprentissage corporel dans un deuxième temps, pour en troisième lieu tenter de rapprocher ces deux perspectives.

\section{Un espace socialisé qui prédispose les démarches corporelles}

En premier lieu donc, il convient d'insister sur ce que la nature a de profondément social, à commencer par la cartographie des groupes qui s'en sont appropriés tout ou partie. Pour reprendre notre exemple lagomorphique, l'adjonction d'un partenaire de chasse comme le chien, et jusqu'au type de chien, modifie radicalement la tournure que prendra la quête. 
Nous entrons ici dans une autre antithèse, formée des deux conséquences à attendre du comportement canin : la fuite ou l'immobilisation du gibier. Aux chiens courants va répondre la fuite des animaux, que ce soit le petit ou le grand gibier, au bois ou en plaine. Dans les plaines françaises où l'on pratique ce type de course, il convient de disposer du droit de chasse sur de vastes étendues. Plutôt que d'être "forcé ", comme dans la battue, le gibier se trouve "mené " à parcourir une longue distance; plus le territoire est vaste et, implicitement, plus grande est l'importance des chasseurs. La différence avec la vénerie va se situer dans la ligne de tireurs postés à proximité d'un passage souvent emprunté et vers lequel poussent les chiens lancés dans une poursuite qui se situe entre la battue et le laissercourre. Dans les zones boisées, renards, sangliers et cervidés subissent la même poursuite, mais cette fois on parlera de " coulée " pour qualifier les chemins de fuite empruntés, leurs poursuivants aux trousses. Victor Scherrer (2002) commente ainsi ce type de course potentiellement génératrice de conflits territoriaux :

"C'est un mode de chasse très convivial et modéré quant au prélèvement ; le plaisir du chasseur réside essentiellement dans la beauté et la gaieté de la poursuite, et dans la musique des chiens (sic.). Mais ce mode de chasse peut être très perturbant pour d'autres espèces de gibier, lorsque les chiens ne sont pas créancés, c'est-à-dire dressés à pister une espèce donnée, ce qui pousse parfois certains grands propriétaires dans les régions où se pratique cette chasse à clore leur territoire d'un grillage » (p.II.26).

A l'opposé, ce n'est absolument pas le comportement que l'on attendra des chiens d'arrêt (braques, épagneuls, pointers, setters, griffons etc.), lesquels ont pour mission et instinct (travaillé) de repérer l'émanation olfactive d'un gibier, de l'approcher en toute discrétion et de lui assigner l'immobilisme, comme pétrifié par le "marquage " que lui impose cette aphasie délibérée. On comprend ici que c'est davantage la disposition des lieux qui rend précieuse cette capacité à fixer la faune, car lorsque la plaine est vaste et le gibier rapide, la chasse peut rapidement se conclure par le renoncement du chasseur, si bien sûr celui-ci ne maîtrise pas le droit de chasse sur un périmètre suffisant. Or, ce fractionnement de l'assise foncière correspond bien aux régions du Nord de la France, où cette méthode ne règne indubitablement pas par hasard. L'arrêt détient encore un autre avantage, beaucoup plus psychologique et valorisant pour le maître, qui n'a à la bouche bien souvent que les expressions les plus emphatiques pour le "travail de son chien ", lequel l'a attendu et fait décoller le gibier au meilleur moment.

A mi chemin entre les courants et les chiens d'arrêt maintenant, les "leveurs " sont de vifs et très ardents springers ou cockers qui ont pour fonction de trouver lapins, lièvres, faisans et bécasses, de les faire se lever, mais sans les poursuivre, afin que le fusil puisse tirer sans risquer de les blesser. Là encore, c'est une caractéristique physique du terrain, à savoir la présence de broussailles, fourrés et ronciers qui font tout l'intérêt de ces leveurs. On comprend aisément que dans ce genre de milieux, l'arrêt n'aurait pour effet que d'éterniser l'attente, le chasseur n'ayant pas les moyens d'accéder facilement à sa proie, alors que la poursuite provoquerait de nombreux accidents sur les chiens dissimulés par la végétation.

On peut conclure de ces usages que l'organisation sociale d'un terroir associée à la parcellisation du foncier, lesquelles conditionnent pour une grande part la possibilité pour les chasseurs de disposer d'un territoire plus ou moins vaste, orientent encore les méthodes retenues pour la pratique. Toujours et encore, la chasse représente un manège qui met en scène la spatialité toute sociologique de la nature. Selon que le droit de chasse d'un groupe 
de pratiquants s'exerce sur un territoire unifié ou a contrario émietté, certaines techniques s'imposent d'elles-mêmes. Pour autant, se maintenir sur cette posture épistémologique holiste, dont la sociologie des années 80 s'est largement faite l'écho (Etudes rurales, 1982), ne permet aucunement de rendre totalement compte des manières dont le corps cynégétique en action actualise son savoir-faire au travers des expériences vécues in situ. Si son ancrage théorique nous informe quant à l'hexis corporelle du chasseur (Kedzierska, 2006), sa façon d'exprimer les valeurs inculquées par ses groupes de référence, il ne dit rien des effets du terrain sur sa manière de réviser son ethos, soit les dispositions éthiques et pratiques communes à un même groupe social.

\section{L'indicible " plaisir » du corps en action}

Pierre Bourdieu l'explicite on ne peut plus clairement : " La force de l'ethos, c'est que c'est une morale devenue hexis, geste, posture » (1992: 133). A l'opposé de ce structuralisme génétique des liens socio-territoriaux, où domine le monde des représentations entre groupes en présence via leur emprise spatiale, une anthropologie du corps propose cette interprétation pragmatique du sens pratique dont les chasseurs font la preuve dans leurs actes concrets. En se rapprochant des sciences cognitives, les spécialistes de l'homme dans sa dimension transversale, tant sur le plan culturel que géographique, établissent le distinguo entre deux types de connaissances: celles verbalisées, bien connues des disciplines du social, et les connaissances procédurales, moins prises en compte. Ces dernières passent par l'intégration physique, comme le fait d'apprendre à marcher dans un champ de labour sans se fouler le pied. Si la première forme de savoir relève de l'instruction, ce qu'on pourrait ramener à la pensée, la seconde en revanche mobilise le corps dans ses multiples facettes : l'aspect sensitif du perçu, le côté moteur de la réaction, la coordination également entre une multitude de muscles. La spécificité de ces procédures, et ce n'est pas la moindre, $c^{\prime}$ est de se tenir très largement dans un stade qui ne nécessite pas la conscience de ce que l'on fait au moment de le faire. En d'autres termes, il n'est pas utile de connaître dans le détail l'ensemble des facultés sollicitées par la parole pour parler, et il serait même paralysant d'y réfléchir à chaque fois que nous émettons un son. C'est en ce sens que les savoirs procéduraux s'enracinent profondément dans le registre du cela va de soi. Le ressenti par rapport à un paysage n'a par exemple pas besoin pour exister de passer par ce processus rationnel. Un inconscient cognitif puissant nous permet de l'apprécier ou au contraire de le détester sans refaire le parcours de la vie d'un œil et des schèmes d'appréciations qui permettent d'émettre un jugement esthétique.

Or, l'anthropologue Jean-Pierre Warnier, qui a fortement contribué à cet axe de réflexion en développant ses travaux sur la culture matérielle (1999), n'a pas manqué de faire le lien entre initiation à la prédation et apprentissage par corps (2009). Faisant référence à des sources aussi diverses dans leurs objets que la danse étudiée par Sylvia Faure (2000) ou le processus de civilisation en Occident chez Norbert Elias (1975), il relève cette étroite analogie :

"En considérant la pratique cynégétique dans un territoire sous l'angle des connaissances procédurales plutôt que des représentations dont elle est l'objet, il est possible de montrer qu'elle mobilise une culture sensori-motrice et matérielle complexe accompagnée d'émotions spécifiques qui s'inscrivent dans la subjectivité du chasseur (p.1). » 
On pourrait étendre cette proximité à d'autres approches, comme celle de la boxe par Loïc Wacquant (2001), la tauromachie vue par Christophe Traïni (2003) ou encore la marche observée chez David Le Breton (2000). Nous avons rencontré la même énigme autour des terrains de jeux de balle tirant leurs règles complexes de la phaeninde des grecs anciens, telle que la confidentielle longue paume en Picardie, les paumistes affirmant avec force l'impossibilité de comprendre les mouvements des équipes sans entrer soi-même dans une formation.

Ce que Warnier cerne parfaitement, c'est également le caractère profondément indicible des "Plaisirs de la chasse", pour reprendre la formule maints fois utilisée par les thuriféraires de la prédation armée et que l'on retrouve dans l'intitulé d'une revue cynégétique contemporaine. A la question de savoir ce qui constituerait l'attraction fondamentale contenue dans cette démarche, le chasseur est bien en mal de fournir une réponse personnelle, se limitant souvent à reproduire la doxa des notables du milieu quant au rôle gestionnaire que la chasse jouerait dans une nature radicalement anthropisée. La logorrhée des porteurs de fusil n'est pourtant pas en cause, bien qu'elle reste sans effet sur le fond d'une motivation devenue suspecte pour nos sociétés: un rapport charnel à la nature, fait d'un ambivalent, mais non contradictoire rapport de domination suprême et de considération respectueuse.

\section{Une nature " bonne à penser »?}

A défaut des représentations sociales, le moteur de cette culture matérielle repose ainsi dans des acquis qui s'incorporent via la répétition gestuelle, à la manière dont s'apprend l'usage d'un vélo par la maîtrise de l'équilibre. II n'est ainsi pas envisageable de simuler l'exercice par un dispositif virtuel: abattre des plateaux d'argile lors d'un ball-trap ne prépare que de très loin au tir en situation réelle, sur un être vivant qui évolue en terrain connu, usant de ses aspérités autant que de l'ensemble des conditions du moment.

Le premier des rabatteurs venus perçoit sans difficulté toute la différence qui sépare le promeneur d'un pisteur avisé. Pour prolonger l'inspiration maussienne, suivre un amateur de lièvre s'apparente à une lecture du topos, à défaut de quoi le cheminement risque fort de passer pour un zigzag ésotérique. Dans la chasse dite "devant soi » au chien d'arrêt, le pratiquant se présente généralement seul à l'entrée d'une plaine retenue pour ses qualités et sa réputation à accueillir le gibier convoité. Seul avec néanmoins l'indispensable compagnon canin, dont le flair sera essentiel pour débusquer l'animal qui se fond aisément dans la couleur d'un labour. Il est véritablement possible à un homme peu expérimenté de passer à quelques dizaines de centimètres sans que ne bronche la proie. II existe indéniablement une initiation oculaire qui passe par un savoir regarder, d'où l'intérêt de cette complicité avec le nez auxiliaire du chien dressé à cet effet. Commence alors pour le porteur de fusil une étrange déambulation, faite de progressions apparemment désordonnées, reproduisant globalement une succession de crochets sans objectif apparent, ponctués d'éphémères haltes. C'est au redémarrage que l'attention se doit d'être maximale, l'animal étant susceptible d'engager sa fuite à cet instant, entamant la plupart du temps une très large trajectoire demi-circulaire, entrecoupées de subites bifurcations. Auparavant son traqueur aura pris soin de repérer les emplacements susceptibles de l'abriter, en fonction de I'humidité ambiante, de la température, mais aussi des conditions climatiques des jours précédents, du dénivelé, des raies de labour plus prononcées entre la fourrière et le reste du champ et moult autres infimes détails. Sans entrer dans tous les méandres quasi savants de 
cette intuition en actes, notons que très rapidement on discerne la matière à des théorisations : sortes de théorèmes que le chasseur fait siens. "Tu vois, là je verrais bien un lièvre remisé. " L'argumentation tourne pourtant court : "Si j'étais un lièvre, je me poserais là, dans ce sillon, aux trois quarts de la pente ; comme ça je verrais arriver de loin et je resterais au soleil, tout en étant invisible. " Arrivés à ce point de l'ascension, un gîte abandonné depuis peu, à peine perceptible au contact visuel mais encore légèrement tiède, vient conforter la prédiction. "Tu vois, je t'avais bien dit. Ca se sent ça. »

S'ensuivent, quelques soient les formes de chasse, des enseignements prenant la tournure de micro philosophies, qui peuvent parfois servir de modèles allégoriques pour orienter son comportement dans la vie sociale. La disparition du lapin de garenne devient le symbole victimisé d'une recherche de productivité agronomique à tous crins et au sein du conseil municipal l'on s'oppose pied à pied au remembrement en cours au nom de la sauvegarde du paysage. Les chasseurs de gibier d'eau sont probablement les plus prolixes dans ce domaine, véhiculant des doctrines sur l'intelligence animale qui dictent leur vision du monde : à la manière des anatidés qui pâtissent de la rétraction des zones humides, ils développent une conception hostile de la société à leur égard, allant jusqu'à générer un syndrome obsidional. Dans leurs cités assiégées donc, les sauvaginiers comme ils s'appellent eux-mêmes, ont constitué des fiefs politiques puissants autour de leur parti : le CPNT, rempart territorial (Bussi, Fourquet, 2002) parfois efficace contre leurs ennemis jurés : les "escrologistes" (sic.).

A partir donc d'une activité aussi anecdotique en apparence que la chasse, en retenant d'elle sa permanence dans les sociétés humaines et à la fois son extrême diversité selon les lieux et les époques, il apparaît une dynamique circulaire entre deux postures : d'une part le corps dans l'espace perçu comme reflet d'une position dans la société, et d'autre part un espace pensé, support de situations dans lesquelles l'acteur physique puise la matière à sa réflexion au travers de son corps. Pour évoquer ces images véhiculées par les chasseurs, comme leur représentation d'une fin annoncée les concernant, conséquence du désamour que leur porterait le reste du corps social, leur posture corporelle les pousse aussi à se considérer comme les " derniers des Mohicans", dans la mesure où ils ressentent physiquement leur " mise en réserve ». Faudrait-il préférer une interprétation de cet état de fait qui se cantonne à considérer la rétraction des biotopes favorables au sauvage comme la simple métaphore du déclin cynégétique ? La nature se décrypte comme un réservoir de signes dans lesquels on peut penser reconnaître des messages ou des préfigurations de l'actualité qui anime les champs sociaux. Pour autant, la disparition de haies bocagères, l'arasement de talus, l'agrandissement du parcellaire ou encore le drainage et l'assèchement de marais sont des observations tangibles. Rien n'interdit d'imaginer la finitude de la chasse à partir de la minéralisation des espaces verts, sans que cela ne vienne contredire le divorce consommé entre les hommes en kaki et une part substantielle de leurs co-sociétaires.

Il importe ainsi de se pencher sur ces territoires "bons à penser », et ce au travers d'une expression langagière centrale pour le corps cynégétique, à savoir le mouvement, ou à défaut la position statique, laquelle revient à la même expressivité par la négative.

\section{Langage spatialisé : la feuille de route du chasseur et ses imprévus}

« Un chasseur c'est d'abord son territoire » relève l'anthropologue Bertrand Hell (1985: 194). Pourtant plus proche d'un culturalisme s'inspirant des flux corporels, il relève avec force 
l'importance du rapport à la spatialité pour le nemrod. Mais cette territorialisation peut ici encore s'interpréter par deux grilles de lecture qui se contredisent l'une l'autre. La première met l'accent sur la dimension politique d'un espace disputé et dans lequel les corps sont contraints par un ordonnancement déjà donné, symbolisant l'ordre en place (Boiral, Brouat, 1984). La seconde grille cherche à réhabiliter la liberté de manœuvre que la subjectivité peut trouver au travers d'un corps qui transforme et produit l'espace, notamment en mobilisant son imaginaire (Ostrowetsky, 1983).

Chez les uns le local n'est que l'expression stratégique d'une idéologie issue de la petite bourgeoisie intellectuelle et culturelle, qui trouve dans le territoire une réponse à la globalisation. La socio-sémioticienne voit en revanche dans le travail des architectesurbanistes en charge des villes nouvelles françaises des années 60 un "imaginaire bâtisseur ". Le raccordement entre ces deux perspectives semble voué à l'échec, bien que l'anthropologie structurale de Claude Lévi-Strauss laisse transparaître une voie en filigrane des hésitations de l'auteur dans l'analyse qu'il fait du village Bororo.

\section{"Je pense, donc je suis... de quelque-part "}

Les chapitres de Tristes tropiques consacrés à l'organisation socio-spatiale des Bororos (1955 : 229-284) fournissent une magistrale démonstration de cette matérialité « bonne à penser ». Rappelons succinctement les traits structurants qui en forment l'armature pour la socio-anthropologie de l'espace, sachant que ce modèle a été suffisamment discuté pour en rester à son noyau dur : l'efficience performative de l'agencement spatial.

Dans les années 30, les populations tribales d'Amazonie parvenaient à reproduire leur stratification sociale en la gravant littéralement dans le plan de leurs villages. Ces derniers étaient organisés sur un mode circulaire, le long d'un cours d'eau, une ligne médiane virtuelle séparant selon un axe parallèle à la rivière la partie de la phratrie tugare de celle des cera. Division binaire capitale, car cette première dichotomie sociale permet de régler le principe exogamique des règles matrimoniales, obligeant les hommes des deux sousgroupes à la résidence matrilocale, dans un système de filiation matrilinéaire. En surface donc les femmes détiennent le pouvoir sur la dévolution des biens matériels et le contrôle du lignage. En revanche la partie immergée de l'iceberg sociographique montre une survalorisation des droits accordés aux hommes dans I'utilisation des ressources naturelles, dont les plumes de volatiles qui permettent de s'embellir par la confection de diadèmes perfectionnés : distinction suprême dans le système de valeurs des bororos qui accordent la primauté à l'esthétique, lui consacrant la majeure partie de leur temps. Par ailleurs, si les huttes d'habitation échappent au masculin, a contrario ce dernier règne sur la Maison des hommes, le Baitemannanage. Pivot du camp, cette Maison des hommes s'ouvre sur la partie cera par la porte tugare, soit celle fréquentée par les habitants des huttes cera issus de la phratrie tugare, et réciproquement sur la partie tugare par la porte cera.

Mais derrière l'apparente égalité entre tugare et cera, Lévi-Strauss le premier s'interroge quant à la prégnance de cette catégorisation, derrière laquelle se profilerait en réalité une autre structuration, triadique celle-là, fondée sur des clans qu'on pourrait qualifier de "supérieurs ", " moyens" et "inférieurs ", auxquels étaient associés des droits exclusifs, dressant un portrait beaucoup plus contrasté des communautés bororos. Ainsi, l'espace villageois socialise aux règles de l'organisation sociale, tout en servant insidieusement un ordre social bien établi. 
Ces considérations spatiaux-organisationnelles ne prennent toute leur portée néanmoins qu'en vis-à-vis de la spiritualité dont se réclament les chasseurs-cueilleurs amazoniens, à savoir une pensée religieuse relevant du totémisme, dont Lévi-Strauss propose une interprétation originale (1962). En se bornant encore une fois aux seules idées directrices, le structuralisme range en deux ordres de réalité d'un côté les groupes humains qui se revendiquent d'un totem, et de l'autre les êtres et les choses totémisés, soit de façon figurée les éléments d'une série culturelle directement en relation avec ceux d'une série naturelle, lesquels servent d'intermédiaire aux premiers pour se classer entre eux. Si donc les bororos se considèrent comme des araras (les perroquets qui offrent les parures les plus recherchées chez eux), leur finalité totémique vise bien à se placer sur l'axe culturel de leurs congénères humains, en mettant en avant les qualités attribuées à leur alter-ego du monde naturel.

Un tel système classificatoire se retrouve chez les adeptes de la sauvagine (Baticle, 2007), I'hexagone présentant un véritable réseau des chasseurs de gibier d'eau, fortement structurés en associations que chapeaute la plus importante d'entre elles en Europe, I'ANCGE. En formant un réseau d'interconnaissance qui passe par la revue officielle de leur regroupement ("La sauvagine »), mais également et de plus en plus souvent via les sites internet spécialisés, les hommes des marais et des grèves (qui s'attribuent des pseudonymes associant faune et territoire : "Colvert.80 » par exemple) font valoir leur emblème, diffusé au travers d'un anatidé fétiche, la plupart de temps symbolique de leur territoire de pratique. Ce marquage permet non seulement de s'identifier dans les nombreuses manifestations revendicatives qui émaillent l'espace public français depuis le début des années 80 , mais il est encore l'objet d'une revendication qui emprunte au chauvinisme micro-régional. A titre de révélateur, La " tête d'Oigne ", à savoir le profil stylisé d'un canard Siffleur, est dressé partout comme la bannière des plus célèbres sauvaginiers, entre 2 et 4000 porteurs de fusils affiliés à la puissante ACDPM de la Baie de Somme, connue depuis 1975 pour rassembler les plus viscéralement acquis à la cause d'un mode de vie se revendiquant en tant que "culture de la nature " (Baticle, 2009). Sur les cantons côtiers du département de la Somme, où l'on compte quelques $8 \%$ de pratiquants au sein de la population, on avance $\mathrm{qu}^{\prime}$ « ici la chasse n'est pas une passion, c'est une religion. »

\section{Le corps pour dépasser le binôme nature versus culture}

Cette emphase doit bien-sûr se comprendre comme une extrapolation stratégique de la " passion ", visant à affirmer la primauté de cette activité au " pays des chasseurs. " Un Jean-Pierre Warnier estimerait vraisemblablement qu'avec un paradigme inspiré de l'analyse structurale l'on en reste au stade de la symbolique des connaissances verbalisées. Le " carré identitaire ", conceptualisé par Sylvia Ostrowetsky (1995) sur le principe du carré sémiotique d'Algirdas Julien Greimas, ne nous aiderait pas davantage à nous situer hors d'une perspective du signe, donc d'un dialogue entre le signifiant et le signifié. Maintenant, l'intellectualisme distant reproché à la théorisation de Lévi-Strauss gagnerait désormais à être revisité à la lumière de la lecture qu'en fait Philippe Descola (2005), pour qui on a beaucoup exagéré la césure taxonomique par laquelle les éléments de la série culturelle ne feraient que jouer des éléments de la série naturelle. Pour l'anthropologue des modes de socialisation de la nature, il existe au contraire dans l'ontologie totémique une indifférenciation très forte entre le monde des humains et celui des non-humains quant à la manière dont ces sociétés établissent un continuum entre les choses et les êtres au sein du vivant. Cette identité de nature posée vaut ici tant sur le plan physique que psychique, 
corporel que spirituel. Autrement dit, pour la pensée totémique, hommes et totems ne se différencient pas, par essence, que ce soit dans leurs "physicalités" ou dans leur intellectualité, au-dehors comme au dedans.

L'avantage fourni par cette perspective qui relativise l'antinomie entre nature et culture, produit de l'anthropocentrisme européen, c'est de réinjecter le corps au centre de l'analyse, à la fois comme enveloppe charnelle et siège de l'intentionnalité. Ici peut intervenir utilement l'anthropologie du corps, en ce qu'elle relie la psyché à la matérialité extérieure.

"Descola estime qu'à tout prendre, la dichotomie occidentale du corps et de l'âme, de la matière et de l'esprit, est une "variante locale" d'une opposition universelle présente dans les vocabulaires du monde entier. On échapperait donc à l'eurocentrisme en se contentant de relâcher le dualisme de l'âme et du corps (ou celui du mental et du physique) en un dualisme du dedans et du dehors (Héran, 2007 : 798). "

A partir de cette critique larvée, le sociologue remarque avec justesse qu'on n'échappe pas aussi facilement au schème binaire cher à la réflexion structurale. On a remplacé le couple nature/culture par un autre: matière/esprit ou corps/âme. Si ce n'est que le binôme proposé se présente sous la forme d'un Janus, le corps représentant autant un contenant qu'un contenu, une matière à la fois placée dans le registre du physique et du psychique, sauf à considérer que les fonctions neurologiques ne seraient pas ancrées également dans de la matière. Cette faculté à assumer les deux faces en même temps correspond à la façon dont les anthropologues du corps définissent les apprentissages procéduraux, si on accepte l'idée que l'incorporation des manières de faire ne sont pas sans effet sur les manières de penser. De plus, bien qu'il s'agisse d'une image encore une fois, l'intérieur et l'extérieur sont affaire de perspective. Je peux vivre mon corps comme extérieur à moi-même, le rejeter en tant que bon représentant de mon identité telle que je me la suis élaborée en pensée, en percevoir des éléments étrangers avec la tumeur, tout comme il m'est possible inversement de ressentir les questionnements de ma psyché dans certaines parties de mon corps, en somatisant. Il y a ainsi avec le corporel un contenu-contenant : une pensée corporelle qui n'est duelle que par abstraction, car la pensée passe par le corps et le corps exprime la pensée. On perçoit par son biais que je suis mal-à-l'aise, tout autant que mon malaise est lié à ce corps dont je ne sais que faire dans cet espace.

Surtout, et dans un second périmètre, la corporéité est un contenu dans un autre contenant : l'environnement justement. Or, c'est par ce dernier que nous réfléchissons, dans la mesure où il nous offre des occasions de questionnements : le soleil se lève à l'Est pour se coucher chaque jour à l'Ouest. Pour revenir ainsi avec la même périodicité, la circularité du soleil autour de la terre est une figure explicative crédible. Afin de renverser la perspective, je dois détacher mon regard de ma corporéité en m'imaginant observer ce corps de la stratosphère. A partir de là il m'est possible d'envisager que c'est mon corps " enterrestré » qui tourne autour du soleil. Mais les exemples de projection peuvent se trouver dans des expériences plus prosaïques.

Enfin, avons-nous besoin que les schémas métaphoriques dont se dotent les sciences soient valides dans les faits pour qu'ils aient une efficience à réfléchir le réel ? Que les métaphores comportent des dangers, comme avec le darwinisme social, n'enlève rien à la pertinence de l'idée générée par le processus de mise en parallèle : il existe aussi des " prédateurs " dans le corps social. La nature peut donc bien être une hypostase de la société, cette société construit également une conception hypostasiée d'elle-même à partir de la nature. Raison 
qui nous pousse à voir chez les chasseurs des entités bien sociales qui s'inspirent aussi de la nature.

\section{Le parcours du corps cynégétique : entre espace, compétition et logique faunistique}

Imbriquer l'incorporation cognitive à la symbolique des lieux nécessiterait de longs développements et impliquerait d'investir de vastes configurations socio-spatiales, qui ne peuvent qu'être évoquées à grands traits dans le cadre de cet article. Dans la perspective que nous tentons de brosser ici, ce rapprochement aurait pour intérêt de s'inscrire dans le débat sur le déterminisme social. Ce champ théorique émergent, sur lequel s'essaient les auteurs réunis par Bruno Maggi (op. cit.), mérite à notre sens que les sciences sociales intègrent les connaissances nouvelles sur le cerveau, autant que sur le fonctionnement corporel. Philippe Perrenoud nous rappelle que la tentative n'est pas si récente.

"Pour Piaget il y a continuité entre l'action sensorimotrice, observable, et l'action mentale qui se constitue progressivement avec l'apparition de la fonction symbolique, et porte non plus sur les objets eux-mêmes, mais sur les signes ou images mentales qui en permettent la représentation (...) La représentation du réel est toujours la résultante d'une activité mentale du sujet épistémique (1976 : 455-456). »

Ce n'est certainement pas céder à l'engouement pour la génétique ou d'autres savoirs développés par les sciences dites "dures " que d'accepter le dialogue en affirmant l'angle d'approche de la socio-anthropologie, de l'économie politique ou de la linguistique. II est possible d'y contribuer sur un axe inspiré par la sémiotique sociologique de l'espace, tout en tenant compte des acquis d'une anthropologie du corps qui intègre les processus de cognition.

Dans le cas de la chasse, instructif parce qu'il s'insère dans des dispositifs qui nécessitent de prendre en compte le comportement de l'animal, le jeu social avec le territoire local peut constituer une porte d'entrée particulièrement riche en enseignements pour expliquer les motivations à s'inscrire dans la pratique. Pour autant, l'attrait en direction de cette sociabilité virile à déambuler s'imprègne au cours d'un long processus exigeant le dépassement du dégoût inspiré tant par la mise à mort, la souffrance animale, que par le dépeçage des cadavres, les odeurs qui en émanent, la vision des tripes jetées aux chiens. C'est en ce sens que Jean-Pierre Warnier voit dans l'initiation précoce le plus puissant moteur de la reproduction sociale du groupe prédateur, justement parce qu'elle rend possible un apprentissage physique du corps à corps homme/animal en amont des imprégnations, désormais socialement dominantes, qui amènent à réprouver la " bestialité » à l'égard du " sauvage ». Or, sur cet aspect de l'initiation cynégétique, la précocité ne se comprend comme facilitatrice à l'adhésion que dans la mesure où elle nous plonge dans un bain familial et localiste qui en dit long sur les manières d'entrer dans la « fraternité des tueurs » (Hell, $1994: 32$ ). 


\begin{tabular}{|l|r|r|}
\hline \multicolumn{1}{|c|}{ Initiateurs } & Nb. cit. & Fréq. \\
\hline Non réponse & 7 & $0,1 \%$ \\
\hline Grands-Parents & 1045 & $19,9 \%$ \\
Oncles/Tantes & 1241 & $23,7 \%$ \\
\hline Père/Mère & 2967 & $56,6 \%$ \\
\hline Frères/Soeurs & 729 & $13,9 \%$ \\
\hline Beaux-Frères & 420 & $8,0 \%$ \\
\hline Cousins & 508 & $9,7 \%$ \\
Époux/Épouse & 64 & $1,2 \%$ \\
\hline Autres liens de Parenté & 218 & $4,2 \%$ \\
\hline Amis d'Enfance & 913 & $17,4 \%$ \\
\hline Voisins & 592 & $11,3 \%$ \\
\hline Collègues de Travail & 322 & $6,1 \%$ \\
\hline Gendres/Belles-Filles & 23 & $0,4 \%$ \\
\hline Seul(e) & 272 & $5,2 \%$ \\
\hline Autres [Précisez SVP : ...... & 214 & $4,1 \%$ \\
\hline TOTAL OBS. & $\mathbf{5 2 4 0}$ & \\
\hline
\end{tabular}

Réponse à choix multiples (Baticle, 2005 : 32).

Ainsi, parmi les 5240 répondants du département de la Somme (Picardie), à savoir 1/5ème des effectifs d'une région connue pour sa cynégéphilie, l'intronisation dans ce rôle passe essentiellement par les ascendants directs et indirects, voire par l'environnement amical et professionnel ou le voisinage. De la sorte, ils ne sont que $5 \%$ à déclarer s'être auto-initiés, sachant que leur première expérience s'est réalisée en moyenne aux alentours de leur dixième année, et ce comme accompagnateur.

Devenus des porteurs de fusils actifs, les manières dont on peut saisir chez eux la réalisation d'un entre-soi microsocial via l'apprentissage par corps de la démarche, réside justement dans le fait des déambulations sur un territoire de chasse qui matérialise la métaphore du corps social. Si l'on retient l'organisation la plus courante dans le Nord de la France, à savoir l'association communale de chasse, on observe qu'il existe non seulement un souci de faire correspondre entité locale et territorialisation de la chasse, mais qui plus est une volonté d'intégrer dans le collectif les postulants qui peuvent faire valoir une appartenance au village, qu'elle soit résidentielle ou liée à la naissance, voire à la primo-socialisation.

Les collectifs (" sociétés de chasse ") qui en découlent rayonnent donc la plupart du temps sur la majeure partie du territoire de la commune au travers des pressions sociales que ressentent les propriétaires du foncier, et à ce titre détenteurs du droit de chasse. Des sociétés qui ont d'autant plus de facilités à réunir les surfaces nécessaires à leur exercice qu'il s'agit de plaines, dont la possession est disséminée entre une multitude de propriétaires. En revanche, pour les zones boisées, ce sont plutôt les petits massifs et les zones tampons, limitrophes des grandes forêts, qui tombent dans leur giron. Sur le terrain, les premières journées de chasse en plaine témoignent plus souvent d'une primauté du groupe, lequel s'impose aux individualités en prescrivant la battue collective. Des groupes en partance de la place communale sont envoyés aux extrémités du territoire afin de rabattre le gibier vers le centre (méthode parfois appelée "le chaudron »). Cette figure spatiale exprime assez bien le sentiment de la collectivité des chasseurs locaux, lesquels considèrent le gibier comme produit de leurs efforts à maintenir les conditions d'une bonne giboyeusité. Ce n'est que dans les semaines suivant "l'ouverture " qu'une plus grande latitude est laissée aux personnalités. Selon le calendrier des espèces autorisées au tir, les coureurs des plaines s'orientent préférentiellement vers un gibier. Pour la perdrix grise par exemple, très commune dans ces régions, de petites équipes se constituent selon les affinités et le voisinage résidentiel dans la commune. Ces groupes restreints vont embrasser le territoire 
en décrivant de larges mouvements qui, dans un premier temps, sont fonction de la connaissance qu'ils détiennent sur la présence des volatiles, mais aussi des habitudes prises à partir du point de départ privilégié. Car ces lignes de fusils vont être amenées à se frotter les unes aux autres, donc à se contourner pour éviter le face-à-face dangereux. Des règles vont ainsi et tacitement se mettre en place, selon la disposition des équipes au moment de la rencontre, leur orientation générale et la configuration des lieux détenus, le tout sous l'influence des animaux poursuivis. Il conviendra en règle générale de renégocier sa stratégie spatiale afin d'éviter de pousser le gibier vers les territoires des sociétés environnantes. De la sorte, on peut percevoir dans le groupe local des hommes en kaki une cohorte de patrouilleurs du territoire qui défend jalousement son gibier à l'encontre des groupes rivaux. Mais les mêmes pourront à l'inverse viser à profiter des erreurs commises par leur voisinage. Quoiqu'il en soit, les critiques fusent dès que ces principes ne sont pas respectés. Paradoxalement, les difficultés commencent sitôt qu'une compagnie de perdrix est prise en chasse, car dès-lors ce sont ses habitudes comportementales qui priment: le vallon emprunté pour se dérober, le boqueteau qui rendra invisible, l'usage du rase-motte. Généralement, les oiseaux s'enfuient alors qu'ils sont encore hors de porté, ce qui induit une technique : poursuivre la compagnie afin de l'éclater en plusieurs sous-groupes et retenir les animaux les moins robustes au vol afin d'espérer les rattraper. Groupés entre approximativement huit et quinze membres, la formation des perdrix n'est fragilisée que divisée (ou lorsqu' on lui assigne la battue collective). Leurs poursuivants se doivent alors de composer avec l'attitude pressentie du gibier, en s'adaptant aux contraintes inhérentes à la physionomie spatiale du droit de chasse, donc à la présence mitoyenne des concurrents, tant interne au groupe territorial que directement adjacente.

Il leur faut encore considérer l'extériorité au sein même du territoire, certaines de ses portions pouvant être " réservées » par des propriétaires désireux de faire valoir un droit de chasse pour eux-mêmes ou pour des "rattindeux " qui auraient échoué à se faire intégrer. La "rattente ", désigne en picard la posture qui consiste à se tenir embusqué sur une position stratégique en attendant de pouvoir profiter du travail de traque des chasses attenantes. Un aplomb rendu possible par la dispersion de la propriété foncière, spécialement dans les bouquets d'arbres isolés au milieu des terres arables, détenus par des propriétaires locaux en rupture de banc ou par des héritiers extérieurs à la sphère locale, échappant ainsi à sa contrainte morale.

\section{Conclusion}

Au travers de ce dernier exemple, se distinguent plusieurs dimensions dans le savoir-être cynégétique. S'impose au regard d'abord des configurations sociales spatialisées, faites de rivalités entre groupes d'acteurs, d'une compétition pour le gibier selon un cadre juridique et coutumier des bonnes manières de faire. Ensuite, l'espace apparaît comme chargé d'une puissance d'évocation symbolique qui fournit des signes évocateurs pour le groupe ou partie de ses membres. C'est pour l'individu une localisation qui joue le rôle de borne évocatrice dans son vécu personnel de chasseur. Les rencontres avec l'animal fondent une cartographie subjective propre à chacun. Mais on retrouve également une sémiotique du territoire avec les déambulations types, comme la principale en régions de plaines qui consiste à réaliser le "tour du territoire." A suivre d'ailleurs les sociologues de l'imaginaire, les circumambulations vaudraient suffisamment le détour pour que l'on s'y attarde, car ces «trajets concentriques sont plus que la simple conscience géographique d'un espace 
approprié : par eux se déroulent les rites des retrouvailles" (Sansot, op. cit. : 57). Pour autant, et au-delà de la rotondité comme forme prise dans le jeu d'une symbolisation culturelle spécifique, de nouvelles perspectives se dessinent quant à la manière dont l'activité symbolique se trouve impactée par une corporation à jouer de l'espace.

" les trajets concentriques permettent l'imprégnation, lente ou rapide, dans un espace et dans les pratiques qu'il autorise et développe. De ce fait, les rencontres qui émaillent ces promenades précisent d'autant mieux les spécificités locales. Le terme promenade implique une caractéristique générale des trajets concentriques : ils se font à pied. Seule la marche offre une vitesse propice à cette sédimentation imaginaire (...) Le lieu tire sa légitimité de lui-même, et non d'une référence à son utilité sur un mode productif. » (Le Breton, op. cit. : 57).

A la manière des circumambulations d'appropriation, portions du corps social local réinscrivant leur exercice prédateur dans l'espace du territoire détenu pour le droit de chasse, il n'est pas étonnant de constater que les chasses, loin de n'être qu'une quête de gibier, sont avant toute chose des démarches inscrites dans les espaces socialisés, et porteurs de socialisation. Jean Jamin (1988) n'hésitait pas, de son côté, à voir dans la tenderie aux grives des ardennais une véritable « éducation sentimentale. "

Ce faisant, les interactions entre corporéité et spatialité trouvent dans la " passion de Diane " deux entrées concordantes : primo l'incorporation des modus operandi nécessaires à l'exercice et secundo l'expressivité des corps virilisés, dans une extériorisation valorisant la résistance physique, mais encore un utopique espoir de faire corps avec la nature. Car audelà de leur volonté de faire de la chasse un réceptacle idéal de leur identification au monde local, les porteurs de fusil laissent apparaître un imaginaire foisonnant, qui emprunte autant à l'espace onirique de l'enfance et de la nostalgie, qu'à une mystique de la ruralité passablement fantasmatique.

En d'autres termes, en se détachant de la finalité explicite qui consisterait uniquement à capter une part du cheptel dont recèle un territoire, on en arrive à questionner d'autres dimensions, plus implicites, qui font de la chasse une manière d'activer son corps dans un périmètre plus ou moins large, visant à exprimer une position légitime dans un espace social défini d'abord par l'appartenance au local. Une lecture de certaines déambulations révélatrices de ces " corps métonymes » (Lupu, 1982), qui par un mode de déplacement ou d'occupation de l'espace rural donnent à voir un statut social, détient l'avantage de ne pas avoir à correspondre à la position occupée dans la société globale.

\section{Bibliographie}

Augoyard J-F. (1979) Essai sur le cheminement quotidien en milieu urbain, Paris : Seuil.

Baticle C. (2005) Les chasseurs de la Somme : portrait social, enquête sociologique en vue du Schéma de gestion cynégétique, Amiens : UPJV.

Baticle C. (2007) Les pratiques de chasse comme affirmations politiques du principe d'autochtonie. Dimensions territoriales des luttes cynégétiques. Etudes de cas de la Picardie, la Normandie et les Pyrénées. Doctorat de sociologie, J. Copans et Bpoint Kalaora (dir.), Amiens : Université de Picardie Jules Verne. 
Baticle C. (2009) "La nature très sociale de l'environnement chez les chasseurs", in M. Dobré, S. Juan (dir.), Consommer autrement. La réforme écologique des modes de vie, Paris: L'Harmattan, pp.199-216.

Baticle C. et Mazzei R. (2011) Habiter la nature au travers de la chasse, documentaire 40', Amiens : ACSÉ.

Boiral P. et Brouat J-P. (1985) "L'émergence de l'idéologie localiste ", in Sociologie du SudEst, $\mathrm{n}^{\circ}$ 41-44 (Identité locale, identité professionnelle), Aix : Université de Provence, pp.35-50.

Bordreuil J-S. et Ostrowetsky S. (1984) "Le social comme sémio-genèse. Eléments de réflexion sur les rapports actuels de la sociologie et de la sémiotique ", in Langage et société, $\mathrm{n}^{\circ} 28$, Paris : $\mathrm{MSH}, \mathrm{pp} .9-36$.

Bourdieu P. (1980) Le sens pratique, Paris : Minuit.

Bourdieu P. (1992) Questions de sociologie, Paris : Minuit.

Bromberger C. et Lenclud G. (1982) « La chasse et la cueillette aujourd'hui. Un champ de recherche anthropologique? ", in Etudes rurales, n'87-88 (La chasse et la cueillette aujourd'hui), Paris : EHESS, pp.7-35.

Bussi M. et Fourquet J. (coord.) (2002) « La mosaïque politique de la France : 15 cartes par canton pour comprendre les élections présidentielles 2002 ", in Cybergéo: http://www.cybergeo.fr, Paris : CNRS-UMR8504.

Chamboredon J-C. (1982) "La diffusion de la chasse et la transformation des usages sociaux de l'espace rural ", in Etudes rurales, ${ }^{\circ} 87-88$ (La chasse et la cueillette aujourd'hui), Paris : EHESS, pp.233-260.

Chamboredon J-C et Lemaire M. (1970) «Proximité spatiale et distance sociale. Les grands ensembles et leur peuplement ", in Revue française de sociologie, vol. XI, ${ }^{\circ} 1$, Paris : Ophrys, pp.3-33.

Dalla Bernardina S. (2011) Retour du prédateur. Mises en scène du sauvage dans la société post-rurale, Rennes : PUR.

Descola P. (2005) Par delà nature et culture, Paris : Gallimard.

Di Meo G. (1991) L'homme, la société, l'espace, Paris : Anthropos.

Elias N. (1975) La civilisation des mœurs, Paris : Calmann-Lévy.

Fabiani J-L. (1982) «Quand la chasse populaire devient un sport. La redéfinition sociale d'un loisir traditionnel ", in Etudes rurales, $n^{\circ} 87-88$ (La chasse et la cueillette aujourd'hui), Paris : EHESS, pp.309-323. 
Faure S. (2000). Apprendre par corps : socio-anthropologie des techniques de danse, Paris: La Dispute.

Foucault M. (1975) Surveiller et punir. Naissance de la prison, Paris : Gallimard.

Hall E. T. (1971) La dimension cachée, Paris : Seuil.

Hell B. (1985) Entre chien et loup : faits et dits de chasse dans la France de l'Est, Paris : MSH.

Hell B. (1994) Le sang noir. Chasse et mythe du sauvage en Europe, Paris : Flammarion.

Héran F. (2007) "Vers une sociologie des relations avec la nature ", in Revue française de sociologie, $\mathrm{n}^{\circ} 4$, Paris : Ophrys, pp.795-806.

Jamin J. (1988) "La tenderie aux grives en Ardennes, une éducation sentimentale ? ", in P. Vannier et D. Meiller (dir.), L'imaginaire de la chasse: hier et demain, Le Creusot: CRC, pp.169-180.

Kedzierska A. (2006) « L'habitus d'un itinérant. Portrait d'un chasseur malinké (Côte d'Ivoire et Mali) ", in I. Sidéra (dir.), La Chasse. Pratiques sociales et symboliques, Paris : De Boccard, pp.45-53.

Lazure M. (1996) Les jeux de balle et de ballon picards: Ballon au Poing, Longue Paume, Balle à la Main, Balle au Tamis, Amiens : CRDP.

Le Breton D. (2000) Eloge de la marche, Paris : Métailié.

Levi-Strauss C. (1955) Tristes tropiques, Paris : Plon.

Levi-Strauss C. (1962) Le totémisme aujourd'hui, Paris : PUF.

Lupu F. (1982) Conférence audiovisuelle sur la lèpre, Nantes.

Lussault M. (2007) L'homme spatial. La construction social de l'espace humain, Paris : Seuil.

Maggi B. (dir.) (2011) Interpréter l'agir : un défi théorique, Paris : PUF.

Ostrowetsky S. (1983) L'imaginaire bâtisseur. Les villes nouvelles françaises, Paris : MéridienKlincksieck.

Ostrowetsky S. (1995) "Les quatre voies de l'identité ", in N. Marouf (dir.), Identité Communauté, Paris : L'Harmattan, pp.23-33.

Perrenoud P. (1976) "De quelques apports piagétiens à une sociologie de la pratique », in Revue européenne des sciences sociales, n³8-39, Genève : Droz, pp.451-470.

Sansot P., Strohl H., Torgue H. et Verdillon C. (1978) L'espace et son double. De la résidence secondaire aux autres formes secondaires de la vie sociale, Paris : Champ urbain. 
Scherrer V. (2002) Réinventer la chasse pour le XXIe siècle, rapport, Paris : CES.

Traïni C. (2003) «Aficionados et opposants à la tauromachie : les formes plurielles de la civilisation ", in Politix, n64 (La question animale), Paris : Lavoisier, pp.103-125.

Wacquant L. (2001) Corps \& âme. Carnets ethnographiques d'un apprenti boxeur, Marseille et Montréal : Agone /Comeau et Nadeau.

Warnier J-P. (1999) Construire la culture matérielle. L'homme qui pensait avec ses doigts, Paris : PUF.

Warnier J-P. (2009) "Chasse et territoires: un apprentissage par corps ", communication écrite, colloque SFER, Clermont-Ferrand, 13 feuillets. 